SNUTP-99-055

\title{
Meson Mass at Large Baryon Chemical Potential in Dense QCD
}

\author{
Deog Ki Hong ${ }^{a}$ ], Taekoon Lee ${ }^{b[}$ and Dong-Pil Min ${ }^{b, c}$ \\ ${ }^{a}$ Department of Physics, Pusan National University, Pusan 609-735, Korea \\ ${ }^{b}$ Center for Theoretical Physics and ${ }^{c}$ Department of Physics, \\ Seoul National University, Seoul 151-742, Korea
}

\begin{abstract}
We reexamine the quark mass induced term in chiral Lagrangian in color-flavor locking phase in dense QCD, and show that the meson mass term is determined by three independent invariants under chiral-axial symmetry, and a meson mass is given in terms of the quark mass, gap, and the chemical potential by $m_{\pi}^{2} \sim$ $m_{q}^{2} \Delta \bar{\Delta} / \mu^{2} \ln \left(\mu^{2} / \Delta^{2}\right)$. Thus mesons become massless as $\mu \rightarrow \infty$.
\end{abstract}

Cold, dense quark system with three massless quark flavors was proposed to be in color-flavor locking phase first by Alford, Rajagopal and Wilczek [G], and subsequently confirmed by others [2] , in which di-quark condensation occurs in a pattern

$$
<\chi_{i}^{a} \chi_{j}^{b}>=-<\bar{\varphi}_{i}^{a} \bar{\varphi}_{j}^{b}>=k_{1} \delta_{i}^{a} \delta_{j}^{b}+k_{2} \delta_{j}^{a} \delta_{i}^{b}
$$

where $\chi_{i}^{a}, \varphi_{i}^{a}$, $a$ the color index, $i$ the flavor index, denote two-component Weyl fermions for the left-handed quarks and the complex conjugate of right-handed quarks, respectively. In this phase the symmetry of dense QCD, namely, the global color-chiral-axial-baryonnumber symmetry $S U_{c}(3) \times S U_{L}(3) \times S U_{R}(3) \times U_{A}(1) \times U_{B}(1)$ is spontaneously broken to

\footnotetext{
${ }^{1}$ Email: dkhong@hyowon.cc.pusan.ac.kr

${ }^{2}$ Email: tlee@ctp.snu.ac.kr

${ }^{3}$ Email: dpmin@phya.snu.ac.kr
} 
$S U_{c+L+R}(3)$ by the quark condensation. The $U_{A}(1)$, which is anomalous at zero density, is a good symmetry at high density, since instanton effects are screened out at large baryon chemical potential $\mu$ [3]. Under the symmetry quarks transform as

$$
\begin{aligned}
& \chi_{i}^{a} \rightarrow U_{L i j} g^{a b} e^{i(\alpha+\beta)} \chi_{j}^{b} \\
& \varphi_{i}^{a} \rightarrow U_{R i j}^{*} g^{* a b} e^{i(\alpha-\beta)} \varphi_{j}^{b}
\end{aligned}
$$

where $U_{L, R} \in S U_{L, R}(3), g \in S U_{c}(3)$ and $e^{i \alpha}, e^{i \beta}$ for the axial and baryon number symmetry, respectively.

Upon the quark condensation $9+9$ Nambu-Goldstone bosons are generated, each 9 coming from the $\chi \chi$ and $\bar{\varphi} \bar{\varphi}$ condensation. In nonlinear realization of the symmetry these Nambu-Goldstone bosons can be represented by the unitary matrices $U_{i}^{a}, V_{i}^{a}$ which parameterize the coset space $S U_{c}(3) \times S U_{L}(3) \times U_{A+B}(1) / S U_{c+L}(3)$ and $S U_{c}(3) \times S U_{R}(3) \times$ $U_{B-A}(1) / S U_{c+R}(3)$, respectively, and transform as

$$
\begin{aligned}
& U_{i}^{a} \rightarrow U_{L i j} g^{a b} U_{j}^{b} e^{i(\alpha+\beta)} \\
& V_{i}^{a} \rightarrow U_{R i j} g^{a b} V_{j}^{b} e^{i(-\alpha+\beta)} .
\end{aligned}
$$

Upon gauging the color symmetry gluons absorb 8 Nambu-Goldstone bosons, becoming massive $m_{A_{\mu}} \sim g F_{\pi}$, where $g$ is the gauge coupling, via Higgs mechanism, and there remain 10 Nambu-Goldstone bosons (mesons) in the physical spectrum. Now when quarks receive small mass via the Dirac mass term

$$
\mathcal{L}_{m_{q}}=-m_{i j} \chi_{i}^{a} \varphi_{j}^{a}+\text { h.c. }
$$

these mesons, except for the one associated with the baryon number symmetry, become massive due to the explicit breaking of the chiral-axial symmetry. Since these mesons would be the lowest lying excitations in cold, dense system such as neutron star their spectrum is of considerable interest and was investigated recently by several groups 四, 河, 6]. A notable feature obtained from the investigation is that at large chemical potential meson mass is independent of the gap, $m_{\pi}^{2} \sim m_{q}^{2}$, where $m_{q}$ denotes quark mass, and when there is no sextet condensation (i.e. $k_{1}=-k_{2}$ ) meson mass is determined by a 
unique chiral-axial invariant and all mesons become massless as any two of the quarks go massless.

In this letter we reexamine this problem in the framework of chiral Lagrangian that includes the quarks as well as the Nambu-Goldstone bosons, and reach to a conclusion different from those obtained from the previous investigations. We find that meson mass is gap-dependent, $m_{\pi}^{2} \sim m_{q}^{2} \Delta \bar{\Delta} / \mu^{2} \ln \left(\mu^{2} / \Delta^{2}\right)$, where $\Delta$ and $\bar{\Delta}$ denote the gaps for particle and antiparticle respectively, and for arbitrary gap parameters $k_{i}$, it is determined by three chiral-axial invariants, and mesons become massless only to the leading order in $\Delta \bar{\Delta} / \mu^{2}$, but massive at next leading order, $m_{\pi}^{2} \sim m_{q}^{2} \Delta^{2} \bar{\Delta}^{2} / \mu^{4} \ln \left(\mu^{2} / \Delta^{2}\right)$, even if there is no sextet condensation and two of the quarks become massless.

We start with the chiral Lagrangian for the mesons. The 9 chiral-axial mesons can be represented by the color singlet field

$$
\Sigma_{i j}=U_{i}^{a} V_{j}^{* a}
$$

and can be described by the Lagrangian

$$
\mathcal{L}_{\Sigma}=\frac{F_{8}^{2}}{4} \operatorname{Tr}\left[\partial_{0} \tilde{\Sigma}^{\dagger} \partial_{0} \tilde{\Sigma}-v^{2} \partial_{i} \tilde{\Sigma}^{\dagger} \partial_{i} \tilde{\Sigma}\right]+\frac{F_{1}^{2}}{2}\left[\left(\partial_{0} \alpha\right)^{2}-v^{2}\left(\partial_{i} \alpha\right)^{2}\right]+\mathcal{L}_{m}(\Sigma),
$$

where we have kept only the leading term in derivative expansion, and factorized the octets $\tilde{\Sigma}$ and $U_{A}(1)$ part by $\Sigma=\tilde{\Sigma} e^{2 i \alpha} . F_{8}, F_{1}$ are the decay constants associated with the octets and the singlet, respectively, and $v$ is the meson velocity which was previously calculated to be $1 / \sqrt{3}\left[4,0\right.$ ]. $\mathcal{L}_{m}(\Sigma)$ is the quark mass induced term, and determines the meson mass. Except for $\mathcal{L}_{m}(\Sigma)$ the Lagrangian is invariant under

$$
\Sigma \rightarrow U_{L} \Sigma U_{R}^{\dagger} e^{2 i \theta}
$$

where $\theta$ is an arbitrary phase, and since the quark mass term (4) is also invariant under the chiral-axial symmetry when the Dirac mass transforms as

$$
m \rightarrow U_{L}^{*} m U_{R}^{t} e^{-2 i \theta},
$$

$\mathcal{L}_{m}(\Sigma)$ should be invariant under the combined transformation (7) and (8). 
$\mathcal{L}_{m}(\Sigma)$ can be expanded in powers of the quark mass matrix. Hereafter we mean by quark mass the Dirac mass, not the Majorana mass induced by the gap. Unlike in zero density QCD where $\mathcal{L}_{m}(\Sigma)$ is linear in the quark mass matrix, the leading term is quadratic in $m_{i j}$ due to the absence of left-right quark condensation. It is not difficult to see that there are three independent terms which are quadratic in quark mass and invariant under (7) and (8), and thus $\mathcal{L}_{m}(\Sigma)$ is given by

$$
\mathcal{L}_{m}(\Sigma)=A\left[\operatorname{Tr}\left(m^{t} \Sigma\right)\right]^{2}+B \operatorname{Tr}\left[\left(m^{t} \Sigma\right)^{2}\right]+C \operatorname{Tr}\left(m^{t} \Sigma\right) \operatorname{Tr}\left(m^{*} \Sigma^{\dagger}\right)+\text { h.c. }
$$

where $A, B$ and $C$ are constants that possibly depend on the gap and the chemical potential.

A convenient framework to determine these coefficients is provided by the following chiral Lagrangian, which is valid at energies below the scale at which the quark condensation occurs, for the quarks and Nambu-Goldstone bosons before color is gauged

$$
\begin{aligned}
\mathcal{L}= & i \bar{\chi}_{i}^{a} \bar{\sigma}^{\nu} \partial_{\nu} \chi_{i}^{a}+\mu \bar{\chi}_{i}^{a} \bar{\sigma}^{0} \chi_{i}^{a}+i \bar{\varphi}_{i}^{a} \bar{\sigma}^{\nu} \partial_{\nu} \varphi_{i}^{a}-\mu \bar{\varphi}_{i}^{a} \bar{\sigma}^{0} \varphi_{i}^{a}-\left[m_{i j} \chi_{i}^{a} \varphi_{j}^{a}+\text { h.c. }\right] \\
& +\left[\chi_{i}^{a}\left(\Delta_{\chi}^{\dagger}\right)_{i j}^{a b} \chi_{j}^{b}-\varphi_{i}^{a}\left(\Delta_{\varphi}\right)_{i j}^{a b} \varphi_{j}^{b}+\text { h.c. }\right]+\mathcal{L}_{\mathrm{NG}}(U, V) .
\end{aligned}
$$

Here,

$$
\begin{aligned}
\left(\Delta_{\chi}^{\dagger}\right)_{i j}^{a b} & =\left(k_{1} U_{i}^{* a} U_{j}^{* b}+k_{2} U_{j}^{* a} U_{i}^{* b}\right) \mathbf{P}_{-}(\partial)+\left(\bar{k}_{1} U_{i}^{* a} U_{j}^{* b}+\bar{k}_{2} U_{j}^{* a} U_{i}^{* b}\right) \mathbf{P}_{+}(\partial) \\
\left(\Delta_{\varphi}\right)_{i j}^{a b} & =\left(k_{1} V_{i}^{a} V_{j}^{b}+k_{2} V_{j}^{a} V_{i}^{b}\right) \mathbf{P}_{+}(\partial)+\left(\bar{k}_{1} V_{i}^{a} V_{j}^{b}+\bar{k}_{2} V_{j}^{a} V_{i}^{b}\right) \mathbf{P}_{-}(\partial),
\end{aligned}
$$

where the projection operators $\mathbf{P}_{\mp}(\partial)$ are given by

$$
\mathbf{P}_{-}(\partial)=(1+i \vec{\sigma} \cdot \hat{\partial}) / 2 \quad \mathbf{P}_{+}(\partial)=(1-i \vec{\sigma} \cdot \hat{\partial}) / 2
$$

and $\bar{k}_{i}$ denote the gap for the antiparticles [7]. $\mathcal{L}_{\mathrm{NG}}(U, V)$, which is irrelevant for our immediate discussion, is the usual chiral Lagrangian for the Nambu-Goldstone bosons alone [8, 9], and also supposed to contain the mass term $\mathcal{L}_{m}(\Sigma)$. Note that $\varphi$ has negative chemical potential, as it should, since $\varphi$ carries negative baryon number.

In this effective Lagrangian the meson mass term $\mathcal{L}_{m}(\Sigma)$ can be obtained by integrating out quarks in the constant background of $U$ and $V$, which gives the one loop quark vacuum energy. This may appear confusing, since the meson mass term is already present in the 
Lagrangian. To avoid the confusion we must regard the effective Lagrangian (10) in Wilsonian sense, namely, that it is obtained by integrating out higher frequency modes to the scale where it is defined. When we integrate out higher frequency modes further to obtain a Lagrangian defined at a lower scale, the renormalization to the meson mass term comes in leading order from the quark vacuum energy, and in this sense the meson mass term can be obtained by integrating out quarks from the UV cutoff $(\sim \mu)$ to the scale of interest, which in this case is the gap.

Now power expansion in quark mass of the quark vacuum energy corresponds to insertions of quark mass in the one loop bubble. Since there is no quark propagator connecting the left-handed quark $(\chi)$ to the right-handed one $(\varphi)$ at zero quark mass, only even number of quark mass insertions are possible. This immediately shows that the leading term in the meson mass must be quadratic in quark mass, as already pointed out in $[1]$.

A straightforward calculation shows that $\mathcal{L}_{m}(\Sigma)$ at two mass insertions is given in the form (9), with the coefficients given as the sum of integrals like

$$
i \int \frac{d^{4} p}{(2 \pi)^{4}} \frac{\Delta \bar{\Delta}}{\left[p_{0}^{2}-\epsilon_{-}^{2}(p)\right]\left[p_{0}^{2}-\epsilon_{+}^{2}(p)\right]}
$$

for $A$ and $B$, and

$$
i \int \frac{d^{4} p}{(2 \pi)^{4}} \frac{\left(p_{0}-\mu\right)^{2}-|\vec{p}|^{2}}{\left[p_{0}^{2}-\epsilon_{-}^{2}(p)\right]\left[p_{0}^{2}-\epsilon_{+}^{2}(p)\right]}
$$

for $C$. Here

$$
\epsilon_{-}^{2}(p)=(|\vec{p}|-\mu)^{2}+\Delta^{2}, \quad \epsilon_{+}^{2}(p)=(|\vec{p}|+\mu)^{2}+\bar{\Delta}^{2}
$$

and we denoted quantities proportional to the particle gap $k_{i}$ by the generic gap $\Delta$ and those for the antiparticle gap $\bar{k}_{i}$ by $\bar{\Delta}$. An interesting feature of the integrands in (13), (14) is that when the quark momentum in the loop is near the Fermi surface $(|\vec{p}| \sim \mu)$, only one quark propagator assumes its Fermi surface value but the other does not. This can be easily understood when we observe that the quark mass term (1) creates a particle and a Dirac hole out of the perturbative vacuum (which is the state in which free massless quarks are filled up to the Fermi surface), and thus when the particle is near the Fermi surface, the Dirac hole is $2 \mu$ off from it. 
Performing the integration, the details of which will be given elsewhere [10], we obtain

$$
A \sim B \sim \Delta \bar{\Delta} \ln \left(\mu^{2} / \Delta^{2}\right), \quad C \sim\left(\Delta^{2} \bar{\Delta}^{2} / \mu^{2}\right) \ln \left(\mu^{2} / \Delta^{2}\right) .
$$

It is interesting to compare this result with the previous calculations. If we assume no sextet condensation, that is, $k_{1}=-k_{2}$, we get $A=-B+O\left(\Delta^{2} \bar{\Delta}^{2} / \mu^{2}\right)$, and thus

$$
\mathcal{L}_{m}(\Sigma) \sim \Delta \bar{\Delta} \ln \left(\mu^{2} / \Delta^{2}\right)\left[\operatorname{det}(m \Sigma) \operatorname{Tr}\left[m^{-1} \Sigma^{*}\right]+\text { h.c. }\right]+O\left[\left(\Delta^{2} \bar{\Delta}^{2} / \mu^{2}\right) \ln \left(\mu^{2} / \Delta^{2}\right)\right]
$$

The leading term in $\Delta \bar{\Delta} / \mu^{2}$, which vanishes if any two of the quarks become massless, is what was found in [四], except for the prefactor, which in their calculation was found to be $\sim \mu^{2}$ instead of our $\sim \Delta \bar{\Delta} \ln \left(\mu^{2} / \Delta^{2}\right.$ ) (To obtain the form in the reference, one must replace $m \rightarrow m^{*}$ and do a field redefinition $\operatorname{det}\left(\Sigma^{*}\right) \Sigma \rightarrow \Sigma$ to compensate the differences in definition). This also shows that, contrary to the claim in 4 , the leading term is not the unique one allowed by the chiral-axial symmetry, and even if two of the quarks become massless the mesons can be massive at next leading order in $\Delta \bar{\Delta} / \mu^{2}$. We also notice that the meson mass term discussed in [6], which is in non-analytic form in the quark mass, does not appear. From the discussion so far, it is clear that such term cannot be present in the effective Lagrangian.

Eq. (16) also implies an interesting behavior for the meson mass at large chemical potential. From (9) we see $m_{\pi}^{2} \sim m_{q}^{2} \Delta \bar{\Delta} \ln \left(\mu^{2} / \Delta^{2}\right) / F_{\pi}^{2}$, where $F_{\pi}$ is the pion decay constant, which can be calculated also within the effective Lagrangian (10). The matrix element in the definition of the decay constant associated with a current

$$
<0\left|J_{\mu}(0)\right| \pi(p)>=i F_{\pi} p_{\mu}
$$

can be evaluated in one loop using the quark-quark-Nambu-Goldstone boson vertices given in (10). Performing the loop calculation we find $F_{\pi} \sim \mu$, in agreement with the

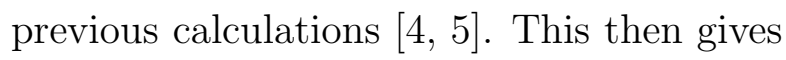

$$
m_{\pi}^{2} \sim m_{q}^{2} \Delta \bar{\Delta} \ln \left(\mu^{2} / \Delta^{2}\right) / \mu^{2}
$$

which shows that the mesons become massless at asymptotically large chemical potential. 
Acknowledgments: This work was supported in part by the Korea Science and Engineering Foundation (KOSEF).

\section{References}

[1] M. Alford, K. Rajagopal, and F. Wilczek, Nucl. Phys. B 537 (1999) 443.

[2] D. K. Hong, hep-ph/9905523; T. Schäfer, hep-ph/9909574; N. Evans, J. Hormuzdiar, S. D. H. Hsu and M. Schwetz, hep-ph/9910313.

[3] R. D. Pisarski and D. H. Rischke, Phys. Rev. Lett. 83 (1999) 37; R. Rapp, T. Schäfer, E.V. Shuryak and M. Velkovsky, hep-ph/9904353.

[4] D.T. Son and M.A. Stephanov, hep-ph/9910491.

[5] M. Rho, A. Wirzba, and I. Zahed, hep-ph/9910550: After submission of our paper, a revised version of this reference has appeared in which it was claimed that mesons remain massless to order $m_{q}^{2}$.

[6] R. Casalbuoni and R. Gatto, hep-ph/9911223.

[7] T. Schäfer and F. Wilczek, Phys. Rev. D 60 (1999) 114033.

[8] D. K. Hong, M. Rho, and I. Zahed, Phys. Lett. B 468 (1999) 261, hep-ph/9906551.

[9] R. Casalbuoni and R. Gatto, Phys. Lett. B 464 (1999) 111, hep-ph/9908227.

[10] D. K. Hong, T. Lee and D.-P. Min, in preparation. 